\title{
Effects of heated water-based exercise on blood pressure: a systematic review
}

\author{
Efeito do exercício em piscina aquecida sobre a \\ pressão arterial: uma revisão sistemática
}

Efecto del ejercicio en piscina climatizada sobre la presión arterial: una revisión sistemática

\section{Awassi Yuphiwa Ngomane ${ }^{[a]}$, Raphael Martins de Abreu ${ }^{[b]}$, Emmanuel Gomes Ciolac ${ }^{[a]^{*}}$}

[a] Universidade Estadual Paulista (Unesp), Bauru, SP, Brazil

[b] Universidade Federal de São Carlos (UFSCar), São Carlos, SP, Brazil

\begin{abstract}
Introduction: Systemic arterial hypertension is one of the main cardiovascular risk factors affecting several population. In this context, heated water-based exercise has emerged as a potential alternative to landbased physical exercise to reduce blood pressure (BP) in hypertensive patients. Objective: To systematically synthesize evidence for the lowering effects of heated water-based exercise on BP in a non-specific population.
\end{abstract}

\footnotetext{
*AYN: Master's student, e-mail: awassiyn@gmail.com

RMA: Doctoral student, e-mail: raphaelmartins.abreu@yahoo.com.br

EGC: PhD, e-mail: ciolac@fc.unesp.br
} 
Methods: Scielo, Pubmed and Scopus electronic databases were searched for studies from 2005 to 2016, with the following descriptors in English: "blood pressure, exercise, immersion, blood pressure and hydrotherapy". A total of 10,461 articles were found and, after applying the inclusion and exclusion criteria, 13 articles were selected and included in the final analysis. All included articles evaluated individuals from different populations and age groups, submitted to a heated water-based exercise session and/or program. Results: The results suggest that both an acute single session and chronic training period (12 to 24 weeks) of heated water-based exercise may reduce BP in different populations (normotensive, hypertensive, postmenopausal women, and heart transplant populations). The magnitude and duration of acute and chronic hypotensive effect of exercise ranged substantially, which was probably due to the variety of exercise frequency, duration and intensity, as well as due to the studied population. Conclusion: These results suggest that heated water-based exercise may promote acute and chronic hypotensive effects in different populations. However, there is no homogeneity in the protocols used, which may have led to the heterogeneity in magnitude and duration of BP reductions.

Keywords: Blood Pressure. Exercise. Hydrotherapy. Hypertension. Immersion.

\section{Resumo}

Introdução: A hipertensão arterial sistêmica (HAS) é um dos principais fatores de risco cardiovasculares que afeta diferentes populações. Sendo assim, o exercício físico em piscina aquecida tem surgido como uma potencial alternativa ao exercício físico em solo para a redução da pressão arterial (PA) de indivíduos hipertensos. Objetivo: Revisar a evidência dos efeitos do exercício físico em piscina aquecida na redução da PA em populações não especificas. Métodos: Foram pesquisadas as bases de dados eletrônicas Scielo, Pubmed e Scopus, de 2005 a 2016, com os seguintes descritores em inglês: "pressão arterial, exercício, imersão, pressão arterial e hidroterapia". Foram encontrados 10.461 artigos e, após a aplicação dos critérios de inclusão e exclusão, foram selecionados 13 artigos que fizeram parte da análise final. Todos os artigos incluídos avaliaram indivíduos de diferentes populações, em diferentes faixas etárias, submetidos a uma sessão e/ou programa de exercícios físicos em piscina aquecida. Resultados: Os resultados sugerem que uma sessão aguda de exercício físico em piscina aquecida, bem como um programa de treinamento de 12 a 24 semanas, pode reduzir a pressão arterial em diferentes populações (normotensos, hipertensos, mulheres na pós-menopausa e transplantados cardíacos). Houve uma grande variação na magnitude e duração do efeito hipotensivo do exercício, o que pode ter sido devido à grande variação de frequência, duração e intensidade das sessões, bem como de populações estudadas. Conclusão: Estes resultados sugerem que o exercício físico em piscina aquecida pode ter efeito hipotensivo agudo e crônico em diferentes populações. No entanto, não há homogeneidade nos protocolos utilizados, o que pode ter levado à heterogeneidade na magnitude e duração das reduções de PA.

Palavras-chave: Pressão Arterial. Exercício. Hidroterapia. Hipertensão. Imersão.

\section{Resumen}

Introducción: La hipertensión arterial sistémica es uno de los principales factores de riesgo cardiovascular que afecta diferentes poblaciones. Siendo así, el ejercicio físico en piscina calentada ha surgido como una alternativa potencial al ejercicio físico en suelo para la reducción de la presión arterial (PA) de pacientes hipertensos. Objetivo: Revisar la evidencia de los efectos del ejercicio físico en la piscina calentada en la reducción de la PA en poblaciones no específicas. Métodos: Buscamos las bases de datos electrónicas Scielo, Pubmed y Scopus, de 2005 a 2016, con los siguientes descriptores en inglés: "presión arterial, ejercicio, inmersión, presión arterial e hidroterapia". Se encontraron 10461 artículos y, después de la aplicación de los criterios de inclusión y exclusión, fueron seleccionados 13 artículos que fueron parte del análisis final. Todos los artículos incluidos evaluaron individuos de diferentes poblaciones en diferentes grupos de edad sometidos a programas de ejercicios físicos acuáticos. Resultados: Los resultados sugieren que el ejercicio físico realizado en una piscina calentada puede llevar a diferentes respuestas en la presión arterial, dependiendo de la frecuencia, la duración y la intensidad de 
las sesiones. Conclusión: Estos resultados sugieren que el ejercicio realizado en una piscina calentada durante 12 a 24 semanas de entrenamiento puede promover efectos benéficos sobre la reducción de la PA. Por otra parte, la sesión aguda no es suficiente para causar un efecto hipotensor. Sin embargo, no hay homogeneidad en los protocolos utilizados, lo que puede haber llevado la divergencia en los resultados.

Palabras clave: Presión Arterial. Ejercicio. Hidroterapia. Hipertensión. Inmersión.

\section{Introduction}

Systemic arterial hypertension (HPT) is a highly prevalent disease associated with high risk for cardiovascular morbidity and mortality [1-3]. However, it is well known that cardiovascular disease risk may be reduced by physical exercise, which promote acute [4-6] and chronic [7-11] reductions on blood pressure (BP), and improves several mechanisms involved in HPT pathophysiology $[8,10,12,13]$. Therefore, regular practice of physical exercise has been recommended for the prevention and management of HPT $[1,2,14]$.

Moderate intensity land-based exercise (LEx) training (i.e. walking, cycling, running and resistance exercise) is commonly recommended for hypertensive patients $[9,11,13,14]$. However, heated water-based exercise (HEx) has been shown to promote important cardiovascular and muscular benefits in different populations [15-19]. For example, substantial BP reduction was found after 2 weeks (nearly $12 / 8 \mathrm{mmHg}$ reduction on systolic/diastolic BP) [17] and 12 weeks (nearly $36 / 12 \mathrm{mmHg}$ reduction on systolic/diastolic BP) [18] of HEx training in subjects with resistant systemic arterial hypertension, which were greater than the BP reductions observed after a LEx training of similar dose (duration, volume, frequency and intensity) [20].

In addition, the buoyancy effect during HEx reduces loading, facilitating the performance of individuals unable to perform high-impact dynamic exercises [16]. However, there is no consensus in literature on which HEx protocol is more appropriate to promote reductions in $\mathrm{BP}$, since the depth of water immersion, exercise intensity and modality, water temperature, and body position during exercise [21-23] can influence the cardiovascular responses. Moreover, although there are systematic reviews and meta-analyses about the acute and chronic effects of LEx on BP [9, 11, 24, 25], none of them have distinguished the effects of HEx. Thus, the aim of this review is to systematically synthesize evidence for the lowering effects of HEx on BP in non-specific population.

\section{Methods}

\section{Search strategy and study selection}

PubMed (via National Library of Medicine), Scopus (Elsevier) and SciELO (Scientific Electronic Library Online) databases were searched for articles about the effects of HEx on BP. The following MeSH terms or keywords were used for the intervention type (immersion AND immersion exercise AND hydrotherapy AND aquatic exercise) and outcomes (blood pressure OR hypertension). The reference list of original articles within this field of study was also reviewed to identify potential eligible trials.

The present systematic review included only randomized clinical trials (RCTs) published between January 2005 and December 2016 that investigated the effects of HEx on BP in adults (age $\geq 18$ years) with or without comorbidities. The inclusion of studies with hypertensive and normotensive individuals was in order to assess the hypotensive effect of HEx and its potential use as a therapeutic and preventive strategy for HPT. Reviews, short communications, letters, case studies, guidelines, theses, dissertations, qualitative studies, scientific conference abstracts, studies on animals, methodological or observational (descriptive only) articles, studies with HEx associated with other therapeutic interventions and clinical trials that did not have the full text available in English or Portuguese language were not included. Two independent reviewers (A.Y. N. and R. M. A.) performed the literature search and study assessment. The articles were initially screened based on their title and abstract, and were selected for full-text screening when any of the reviewers considered the abstract potentially eligible. 


\section{Data extraction and analysis}

Data on study source, sample size, participants characteristics (i.e. age, sex, baseline BP and physical activity levels, comorbidities), method used to measure BP, characteristics of HEx (i.e. type, frequency, intensity and duration of exercise; water temperature; depth of water immersion), LEx (i.e. type, frequency, intensity and duration of exercise) and control interventions (i.e. type, frequency and duration), outcomes and limitations of the studies included were extracted independently by two authors (A. Y. N. and R. M. A.). The two reviewers also independently assessed methodological quality of included studies usingthe Physiotherapy Evidence Database(PEDro) scale ${ }^{1}$. When there were disagreements between reviewers on data extraction, data analyses and/or quality assessment, athird reviewer (E. G. C.) was consulted to solve the discrepancy. The results of thesystematic review are presented descriptively (e.g.means, standard deviations, and minimum and maximum values).

\section{Results}

The electronic database search identified 10,461 articles. A total of 54 duplicate articles were removed, and 103,58 articles were excluded after screening by title and abstract. Full-text screening for eligibility of the remaining 49 articles removed 23 non-randomized clinical trials, five articles published before January 2005, six articles who did not present BP outcomes for all interventions, and two reviews. Finally, 13 clinical trials assessing acute (BP response to a single HEx session) $[19,21,23,26-28]$ or chronic (BP response to a HEx training program) [17, 18, 29-33] effect of HEx on BP were included in the present review (Figure 1).

\section{Study and subject characteristics}

General characteristics of each study included in the present review are displayed in Table 1 . Six studies with sample size ranging from 14 to 20 subjects assessed the acute effect of HEx on BP in physically inactive hypertensive [21, 26, 28] and normotensive [23, 26] individuals, recreationally active normotensive [27], and sedentary heart transplant recipients [6], totaling 105 participants. Two studies included only young individuals $[23,27]$, two studies included only middle-aged individuals $[26,28]$ and one study included both middleaged and older individuals [21]. All studies assessing acute effect of HEx on BP had randomized cross-over design with one $[6,21,23,27,28]$ or two groups [26], and assessed the effect of HEx versus LEx session [23, 27], control session (CON) [28] or both LEx and CON [6, 21, 26]. One study further compared the BP response to HEx between hypertensive and normotensive subjects [26].

Six RCTs with an initial sample size ranging from 26 to 60 subjects assessed the chronic effect of HEx on BP in normotensive [30], pre-hypertensive [32] and hypertensive $[29,31]$ individuals, in patients with resistant hypertension
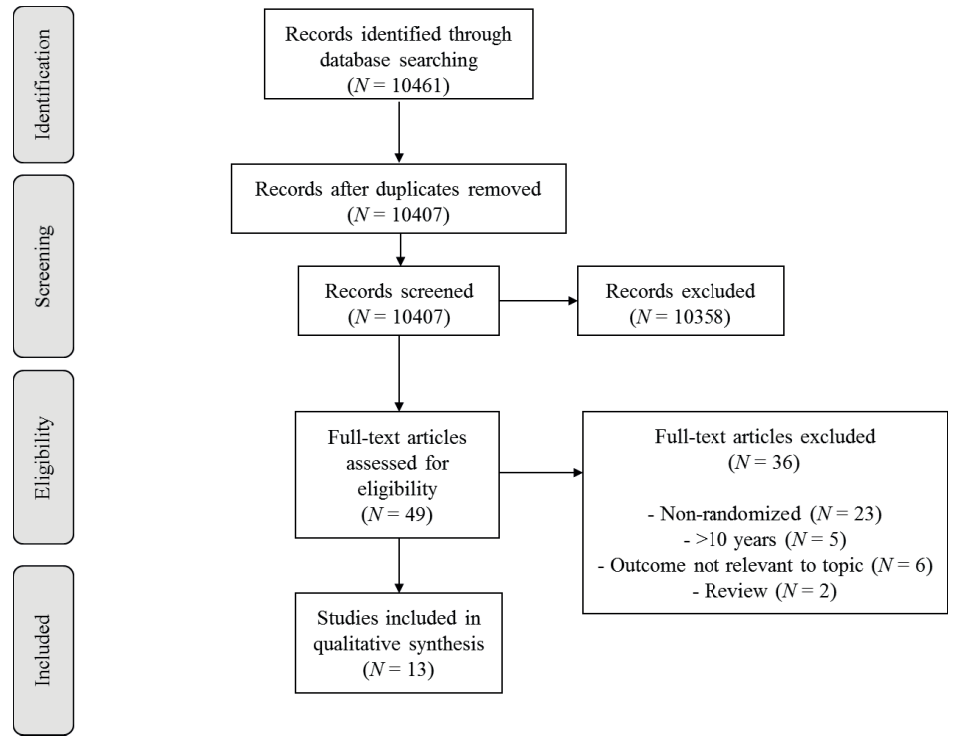

Figure 1 - Flowchart of the process of screening and selection of articles for inclusion in the review.

1 See <http://www.pedro.org.au>. 
$[17,18]$, and in postmenopausal women [33], totaling 292 randomized participants. Six studies included only middleaged subjects [17, 18, 30-33] and one study included only older subjects [29]. All chronic studies had parallel design and assessed the effect of HEx versus LEx [30], CON [17, 18, $31,32]$ or both LEx and CON $[29,33]$.
Finally, the study quality using PEDro-scale is shown in Table 2. The median PEDro score of studies assessing the acute effect of HEx on BP was 6, with a range from 3 to 8 . In the studies assessing the chronic effect of HEx on BP, the median PEDro score was 7, with a range from 5 to 9 .

Table 1 - Characteristics of included studies

(To be continued)

\begin{tabular}{|c|c|c|c|c|c|c|}
\hline $\begin{array}{l}\text { First author } \\
\text { (Country) / } \\
\text { Design } \\
\end{array}$ & $\begin{array}{l}\text { Population } \\
\text { assessed }\end{array}$ & HEx intervention & LEx intervention & $\begin{array}{l}\text { Control } \\
\text { intervention }\end{array}$ & $\begin{array}{l}\text { Water } \\
\text { temperature }\end{array}$ & $\begin{array}{l}\text { BP and secondary } \\
\text { outcomes assessment }\end{array}$ \\
\hline \multicolumn{7}{|c|}{ Studies assessing acute response to HEx } \\
\hline $\begin{array}{l}\text { Cunha et al. } \\
\text { (Brazil) / } \\
\text { cross-over [28] }\end{array}$ & $\begin{array}{l}18 \text { overweight } \\
\text { (mean age } \\
54.4 \pm 7.9 \\
\text { yrs.) and obese } \\
\text { hypertensive } \\
\text { women (mean } \\
\text { age } 56.4 \pm 6.6 \\
\text { yrs.) were } \\
\text { randomly } \\
\text { assigned to } \\
\text { HEx and CON } \\
\text { intervention. }\end{array}$ & $\begin{array}{l}\text { The water } \\
\text { aerobics } \\
\text { exercise session } \\
\text { consisted of } \\
\text { a } 45 \text {-minute } \\
\text { training at the } \\
\text { intensity of } \\
70 \%-75 \% \text { of } \\
\text { maximum heart } \\
\text { rate adjusted } \\
\text { for the aquatic } \\
\text { environment. }\end{array}$ & NR & $\begin{array}{l}\text { The control } \\
\text { group did not } \\
\text { enter the pool } \\
\text { and did not } \\
\text { perform any } \\
\text { exercise. }\end{array}$ & $28.5^{\circ} \mathrm{C}$ & $\begin{array}{l}\text { Systolic and diastolic } \\
\text { BP was measured } \\
\text { before, immediately } \\
\text { after, and every } 10 \text { min } \\
\text { up to } 30 \text { min after HEx } \\
\text { and CON intervention, } \\
\text { using a semiautomatic } \\
\text { device (Omron 705- } \\
\text { CP). }\end{array}$ \\
\hline $\begin{array}{l}\text { Castro et al. } \\
\text { (Brazil) / } \\
\text { cross-over [6] }\end{array}$ & $\begin{array}{l}18 \text { heart } \\
\text { transplant } \\
\text { patients ( } 12 \\
\text { male/6 female, } \\
\text { mean age } 45.7 \\
\pm 2.7 \text { yrs.) } \\
\text { were randomly } \\
\text { assigned to HEx, } \\
\text { LEx and CON. }\end{array}$ & $\begin{array}{l}30 \text { min moderate- } \\
\text { intensity (11-13 } \\
\text { in the 6-20 } \\
\text { RPE) aerobic } \\
\text { exercise (walking } \\
\text { inside the pool } \\
\text { immersed up } \\
\text { to the xiphoid } \\
\text { process). }\end{array}$ & $\begin{array}{l}30 \text { min moderate- } \\
\text { intensity (11-13 } \\
\text { in the } 6-20 \\
\text { RPE) aerobic } \\
\text { exercise (walking } \\
\text { on a motorized } \\
\text { treadmill). }\end{array}$ & $\begin{array}{l}30 \text { min of resting } \\
\text { quietly in the } \\
\text { seated position. }\end{array}$ & $30-32{ }^{\circ} \mathrm{C}$ & $\begin{array}{l}\text { Ambulatory BP } \\
\text { (systolic and diastolic) } \\
\text { was measured during } \\
\text { 24h after HEx, LEx } \\
\text { and CON intervention, } \\
\text { using an automatic } \\
\text { device (Dyna-Mapa } \\
\text { ABP monitor). }\end{array}$ \\
\hline $\begin{array}{l}\text { Garzon et al. } \\
\text { (Canada) / } \\
\text { cross-over [23] }\end{array}$ & $\begin{array}{l}20 \text { healthy } \\
\text { normotensive } \\
(18 \text { male/2 } \\
\text { female, mean } \\
\text { age }(32 \pm \\
7 \text { yrs.) were } \\
\text { randomly } \\
\text { assigned to HEx } \\
\text { and LEx. }\end{array}$ & $\begin{array}{l}\text { Pedaling } \\
\text { immersed on } \\
\text { immersible } \\
\text { ergocycle } \\
\text { (Hydrorider } ® \text { )- } \\
\text { (immersion } \\
\text { to the chest } \\
\text { level). External } \\
\text { power output } \\
\text { on immersible } \\
\text { ergocycle was } \\
\text { controlled by } \\
\text { pedaling rate } \\
\text { (rpm). Initial rpm } \\
\text { was set at } 40 \\
\text { rpm and was } \\
\text { increased by } 10 \\
\text { rpm until } 70 \text { rpm } \\
\text { and thereafter } \\
\text { by } 5 \text { rpm until } \\
\text { exhaustion. }\end{array}$ & $\begin{array}{l}\text { Pedaling on } \\
\text { dryland ergocycle } \\
\text { (Ergoline 800S; } \\
\text { Bitz, Germany). } \\
\text { Initial external } \\
\text { power output of } \\
\text { dryland ergocycle } \\
\text { protocol was } \\
\text { set at } 25 W \\
\text { and increased } \\
\text { by } 25 W \text { every } \\
\text { minute until } \\
\text { exhaustion. }\end{array}$ & NR & $30^{\circ} \mathrm{C}$ & $\begin{array}{l}\text { Central hemodynamic } \\
\text { parameters were } \\
\text { measured continuously } \\
\text { during exercise and } \\
\text { a 5-min recovery } \\
\text { period using a } \\
\text { sphygmomanometer } \\
\text { (WelchAllyn, USA). } \\
\text { Central hemodynamic } \\
\text { was measured } \\
\text { continuously } \\
\text { at rest, during } \\
\text { exercise and after } \\
\text { exercise cessation } \\
\text { using impedance } \\
\text { cardiography. }\end{array}$ \\
\hline
\end{tabular}


(To be continued)

\begin{tabular}{|c|c|c|c|c|c|c|}
\hline $\begin{array}{l}\text { First author } \\
\text { (Country) / } \\
\text { Design }\end{array}$ & $\begin{array}{l}\text { Population } \\
\text { assessed }\end{array}$ & HEx intervention & LEx intervention & $\begin{array}{l}\text { Control } \\
\text { intervention }\end{array}$ & $\begin{array}{l}\text { Water } \\
\text { temperature }\end{array}$ & $\begin{array}{l}\text { BP and secondary } \\
\text { outcomes assessment }\end{array}$ \\
\hline \multicolumn{7}{|c|}{ Studies assessing acute response to HEx } \\
\hline $\begin{array}{l}\text { Luza et al. } \\
\text { (Brazil) / } \\
\text { cross-over [26] }\end{array}$ & $\begin{array}{l}8 \text { sedentary } \\
\text { normotensive (1 } \\
\text { male/7 female, } \\
\text { mean age } 62 \\
\pm 3.7 \text { yrs.) and } \\
12 \text { sedentary } \\
\text { hypertensive ( } 3 \\
\text { male/9 female, } \\
\text { mean age } 59 \\
\pm 2.6 \text { yrs.) } \\
\text { were randomly } \\
\text { assigned to HEx, } \\
\text { LEx and CON } \\
\text { intervention. }\end{array}$ & $\begin{array}{l}\text { Heating; aerobic } \\
\text { exercises; } \\
\text { fortification; } \\
\text { And stretching } \\
\text { and relaxation, } \\
\text { lasting } 45 \\
\text { minutes } \\
\text { [moderate- } \\
\text { intensity } \\
\text { (83-85\% of } \\
\text { HRMAX)]. }\end{array}$ & $\begin{array}{l}\text { The exercise } \\
\text { was performed } \\
\text { in athletics } \\
\text { track, where the } \\
\text { subjects were } \\
\text { instructed to walk } \\
\text { for } 45 \text { minutes } \\
\text { in the heart rate } \\
\text { intensity measured } \\
\text { at the intensity } \\
\text { corresponding } \\
\text { to the anaerobic } \\
\text { threshold } \\
\text { [moderate- } \\
\text { intensity (83-85\% } \\
\text { of HRMAX)]. }\end{array}$ & $\begin{array}{l}\text { In the ground } \\
\text { rest protocol, } \\
\text { the participants } \\
\text { remained sitting } \\
\text { for } 45 \text { minutes. } \\
\text { In the rest } \\
\text { protocol in } \\
\text { the water, } \\
\text { participants } \\
\text { were immersed } \\
\text { for } 45 \text { minutes } \\
\text { in the pool. } \\
\text { (The immersion } \\
\text { occurred up to } \\
\text { shoulder height.) }\end{array}$ & $30-32^{\circ} \mathrm{C}$ & $\begin{array}{l}\text { Systolic and diastolic } \\
\text { BP (standard mercury } \\
\text { sphygmomanometer } \\
\text { Sankey), and Heart } \\
\text { Rate were measured } \\
\text { before and after (30, } \\
60 \text { and } 90 \text { min) each } \\
\text { intervention. }\end{array}$ \\
\hline $\begin{array}{l}\text { Pugh et al. } \\
\text { (Australia) / } \\
\text { cross-over [27] }\end{array}$ & $\begin{array}{l}15 \text { recreationally } \\
\text { active healthy } \\
\text { normotensive ( } 8 \\
\text { male/ } 7 \text { female, } \\
\text { mean age } \\
26 \pm 4 \text { yrs.) } \\
\text { were randomly } \\
\text { assigned to } \\
\text { HEx and LEx } \\
\text { intervention. }\end{array}$ & $\begin{array}{l}\text { Once immersed } \\
\text { in water, } \\
\text { participants } \\
\text { remained in the } \\
\text { resting upright } \\
\text { position for } 5 \\
\text { min, which was } \\
\text { followed by a 20- } \\
\text { min bout of low- } \\
\text { intensity exercise } \\
\text { consisting of } \\
\text { a repetitive } \\
\text { stepping protocol } \\
\text { (approximately } \\
100 \text { bpm). Upon } \\
\text { completion of the } \\
\text { exercise bout, } \\
\text { participants } \\
\text { remained in the } \\
\text { resting upright } \\
\text { position for a } \\
\text { further } 5 \text { min, } \\
\text { after which, } \\
\text { the pumps } \\
\text { were reversed } \\
\text { to empty the } \\
\text { tank-30 min low- } \\
\text { intensity (HR = } \\
100 \text { bpm). }\end{array}$ & $\begin{array}{l}\text { Participants } \\
\text { remained in } \\
\text { the upright } \\
\text { standing position } \\
\text { and avoided } \\
\text { movement for } 5 \\
\text { min. This was } \\
\text { followed by a 20- } \\
\text { min bout of low- } \\
\text { intensity exercise } \\
\text { consisting of } \\
\text { a repetitive } \\
\text { stepping protocol } \\
\text { (approximately } \\
\text { 100 bpm). Upon } \\
\text { completion of the } \\
\text { exercise bout, } \\
\text { participants } \\
\text { remained in the } \\
\text { resting upright } \\
\text { position for a } \\
\text { further } 5 \text { min. }\end{array}$ & NR & $30^{\circ} \mathrm{C}$ & $\begin{array}{l}\text { Finometer PRO } \\
\text { (Finapres Medical } \\
\text { Systems, Amsterdam, } \\
\text { the Netherlands) } \\
\text { was used to monitor } \\
\text { relative changes in } \\
\text { mean BP, cardiac } \\
\text { output, and stroke } \\
\text { volume, before, during, } \\
\text { and } 20 \text { min after HEx } \\
\text { and LEx intervention. }\end{array}$ \\
\hline
\end{tabular}


(To be continued)

\begin{tabular}{|c|c|c|c|c|c|c|}
\hline $\begin{array}{l}\text { First author } \\
\text { (Country) / } \\
\text { Design }\end{array}$ & $\begin{array}{l}\text { Population } \\
\text { assessed }\end{array}$ & HEx intervention & LEx intervention & $\begin{array}{l}\text { Control } \\
\text { intervention }\end{array}$ & $\begin{array}{l}\text { Water } \\
\text { temperature }\end{array}$ & $\begin{array}{l}\text { BP and secondary } \\
\text { outcomes assessment }\end{array}$ \\
\hline \multicolumn{7}{|c|}{ Studies assessing acute response to HEx } \\
\hline $\begin{array}{l}\text { Sosner et al. } \\
\text { (Canada) / } \\
\text { cross-over [21] }\end{array}$ & $\begin{array}{l}42 \text { hypertensive } \\
\text { adults ( } 22 \\
\text { male/20 female, } \\
\text { mean age } \\
65 \pm 7 \text { yrs.) } \\
\text { were randomly } \\
\text { assigned to HEx, } \\
\text { LEx and CON } \\
\text { intervention. }\end{array}$ & $\begin{array}{l}30 \text { min High- } \\
\text { intensity interval } \\
\text { exercise in } \\
\text { up-to-the-chest } \\
\text { immersed } \\
\text { condition was } \\
\text { performed on a } \\
\text { mechanically- } \\
\text { braked cycle } \\
\text { ergometer } \\
\text { (Hydrorider, } \\
\text { DIESSE, San } \\
\text { Lazzaro di Savena, } \\
\text { Italia). Each } \\
\text { exercise session } \\
\text { was preceded by } \\
\text { a 5-min warm-up } \\
\text { consisting in } \\
\text { pedaling at } 40 \\
\text { rpm and followed } \\
\text { by a 5-min } \\
\text { passive recovery } \\
\text { period in a sitting } \\
\text { position that began } \\
\text { immediately after } \\
\text { exercise cessation. }\end{array}$ & $\begin{array}{l}\text { Moderate- } \\
\text { intensity } \\
\text { continuous } \\
\text { exercise in } \\
\text { dry land were } \\
\text { performed on a } \\
\text { stationary bicycle } \\
\text { ergometer -- 24- } \\
\text { min at 50\% peak } \\
\text { power output. } \\
\text { High-intensity } \\
\text { interval exercise } \\
\text { in dry land were } \\
\text { performed on a } \\
\text { stationary bicycle } \\
\text { ergometer-- two } \\
\text { sets of } 10-\text { min } \\
\text { with phases } \\
\text { of } 15 \text {-sec } \\
100 \% \text { peak } \\
\text { power output } \\
\text { interspersed by } \\
15 \text {-sec of passive } \\
\text { recovery. }\end{array}$ & NR & $30^{\circ} \mathrm{C}$ & $\begin{array}{l}\text { The 24h Ambulatory } \\
\text { BP (Systolic and } \\
\text { Diastolic) was } \\
\text { measured with a } \\
\text { brachial cuff-based } \\
\text { oscillometric device } \\
\text { Mobil-0-Graph PWA } \\
\text { Monitor (I.E.M. GmbH, } \\
\text { Stolberg, Germany)- } \\
\text { The monitor was } \\
\text { programmed to } \\
\text { measure BP every } 20 \\
\text { min during the overall } \\
24 \mathrm{~h} . \\
\text { Arterial stiffness } \\
\text { was automatically } \\
\text { assessed, using the } \\
\text { same oscillometric } \\
\text { device Mobil-0-Graph } \\
\text { PWA Monitor (I.E.M. } \\
\text { GmbH, Stolberg, } \\
\text { Germany), at rest with } \\
\text { the patient in supine } \\
\text { position in a quiet } \\
\text { atmosphere for the } \\
\text { first measurement, and } \\
\text { every } 20 \text { min during } \\
\text { the following } 24 \mathrm{~h} .\end{array}$ \\
\hline \multicolumn{7}{|c|}{ Studies assessing chronic adaptation to HEx } \\
\hline $\begin{array}{l}\text { Arca et al. } \\
\text { (Brazil) / } \\
\text { Parallel [29] }\end{array}$ & $\begin{array}{l}52 \text { hypertensive } \\
\text { women (mean } \\
\text { age } 64 \pm 7 \\
\text { yrs.) randomly } \\
\text { assigned to HEx } \\
(n=19), \text { LEx } \\
(n=19) \text { or } \\
\text { CON }(n=14) \\
\text { groups. }\end{array}$ & $\begin{array}{l}\text { 50-minute } \\
\text { sessions on a } \\
\text { three-time per } \\
\text { week basis } \\
\text { during } 12 \text { weeks. } \\
\text { The heart rate } \\
\text { was maintained } \\
\text { around } 50 \% \text { to } \\
60 \% \text { of heart } \\
\text { rate reserve. }\end{array}$ & $\begin{array}{l}50 \text {-minute } \\
\text { sessions on a } \\
\text { three-time per } \\
\text { week basis during } \\
12 \text { weeks. Dry } \\
\text { land exercise } \\
\text { was performed in } \\
\text { stationary bikes. } \\
\text { In the land dry } \\
\text { exercise program, } \\
\text { the heart rate was } \\
\text { maintained around } \\
50 \% \text { to } 60 \% \text { of } \\
\text { heart rate reserve. }\end{array}$ & $\begin{array}{l}\text { Did not change } \\
\text { his eating } \\
\text { habits and } \\
\text { remained without } \\
\text { practicing } \\
\text { physical activity } \\
\text { in the period }\end{array}$ & $33-33.5^{\circ} \mathrm{C}$ & $\begin{array}{l}\text { BP measurements were } \\
\text { performed with a mercury } \\
\text { sphygmomanometer } \\
\text { Premium G-tech }{ }^{8} \text {, } \\
\text { adequately calibrated. } \\
\text { In every one of the } 12 \\
\text { weeks of intervention in } \\
\text { aquatic and land, BP and } \\
\text { heart rate were measured } \\
\text { before subjects begun } \\
\text { exercising, so respectively } \\
\text { before immersion in water } \\
\text { or before they had a walk } \\
\text { on land. }\end{array}$ \\
\hline
\end{tabular}


(To be continued)

\begin{tabular}{|c|c|c|c|c|c|c|}
\hline $\begin{array}{l}\text { First author } \\
\text { (Country) / } \\
\text { Design }\end{array}$ & $\begin{array}{l}\text { Population } \\
\text { assessed }\end{array}$ & HEx intervention & LEx intervention & $\begin{array}{l}\text { Control } \\
\text { intervention }\end{array}$ & $\begin{array}{l}\text { Water } \\
\text { temperature }\end{array}$ & $\begin{array}{l}\text { BP and secondary } \\
\text { outcomes assessment }\end{array}$ \\
\hline \multicolumn{7}{|c|}{ Studies assessing chronic adaptation to HEx } \\
\hline $\begin{array}{l}\text { Colado et al. } \\
\text { (Spain) / } \\
\text { Parallel [33] }\end{array}$ & $\begin{array}{l}46 \\
\text { postmenopausal } \\
\text { women } \\
\text { randomly } \\
\text { assigned to HEx } \\
(\mathrm{n}=15 \text {, mean } \\
\text { age } 54.7 \pm 2 \\
\text { yrs.), LEx ( } \mathrm{n}= \\
21, \text { mean age } \\
54 \pm 2.8 \text { yrs.) } \\
\text { or CON ( } \mathrm{n}= \\
10, \text { mean age } \\
52.9 \pm 1.9 \text { yrs.) } \\
\text { groups. }\end{array}$ & $\begin{array}{l}\text { Training was } \\
\text { performed } \\
\text { twice per week } \\
\text { in the first } 12 \\
\text { weeks and } \\
\text { three times per } \\
\text { week for weeks } \\
\text { 13-24. The } \\
\text { total duration } \\
\text { of the training } \\
\text { session ranged } \\
\text { from } 35 \text { to } 60 \\
\text { min depending } \\
\text { on the phase } \\
\text { of periodization } \\
\text { of the training } \\
\text { program. }\end{array}$ & $\begin{array}{l}\text { Training was } \\
\text { performed twice } \\
\text { per week in the } \\
\text { first } 12 \text { weeks } \\
\text { and three times } \\
\text { per week for } \\
\text { weeks } 13-24 . \\
\text { The total duration } \\
\text { of the training } \\
\text { session ranged } \\
\text { from } 35 \text { to } 60 \\
\text { min depending } \\
\text { on the phase } \\
\text { of periodization } \\
\text { of the training } \\
\text { program. }\end{array}$ & $\begin{array}{l}\text { Did not change } \\
\text { his eating } \\
\text { habits and } \\
\text { remained without } \\
\text { practicing } \\
\text { physical activity } \\
\text { in the period. }\end{array}$ & NR & $\begin{array}{l}\text { BP were taken at the } \\
\text { same physician's } \\
\text { office within } 1 \text { week } \\
\text { prior to and after } \\
\text { the physical pre } \\
\text { and post-testing, } \\
\text { respectively. A resting } \\
\text { BP measurement was } \\
\text { taken after } 10 \text { min of } \\
\text { quiet sitting. The BP } \\
\text { measurement was } \\
\text { repeated in the same } \\
\text { manner after } 48 \mathrm{~h} \text { to } \\
\text { ensure reliability. }\end{array}$ \\
\hline $\begin{array}{l}\text { Farahani et al. } \\
\text { (Iran)/ } \\
\text { Parallel [34] }\end{array}$ & $\begin{array}{l}40 \text { men with } \\
\text { stage } 1 \text { or } \\
2 \text { essential } \\
\text { hypertension } \\
\text { were assigned } \\
\text { to HEx ( } n=12 \text {, } \\
\text { mean age } 48.33 \\
\pm 10.74 \text { yrs.) or } \\
\text { CON ( } n=28, \\
\text { mean age } 46.96 \\
\pm 11.58 \text { yrs.) } \\
\text { groups. }\end{array}$ & $\begin{array}{l}\text { Subjects in the } \\
\text { intervention group } \\
\text { participated in } \\
\text { a supervised } \\
10 \text {-week water } \\
\text { aerobic training } \\
\text { program of } 55 \\
\text { min sessions, } 3 \\
\text { days per week on } \\
\text { alternate days. } \\
\text { The exercise } \\
\text { intensity was } \\
\text { set at } 60-65 \% \\
\text { of the maximal } \\
\text { heart rate and } \\
\text { increased } \\
\text { gradually up to } \\
70-75 \% \text { during } \\
\text { the program. }\end{array}$ & NR & $\begin{array}{l}\text { The control } \\
\text { group were not } \\
\text { involved in any } \\
\text { regular training } \\
\text { program during } \\
\text { this period. }\end{array}$ & $31-32^{\circ} \mathrm{C}$ & $\begin{array}{l}\text { Blood pressures } \\
\text { were measured } \\
\text { using mercury } \\
\text { sphygmomanometers } \\
\text { (Richter, Germany). } \\
\text { Blood pressure was } \\
\text { measured at baseline } \\
\text { and } 48 \text { hours after the } \\
\text { last exercise session } \\
\text { to avoid the acute } \\
\text { effects of a single bout } \\
\text { of exercise. }\end{array}$ \\
\hline $\begin{array}{l}\text { Guimarães et al. } \\
\text { (Brazil) / } \\
\text { Parallel [18] }\end{array}$ & $\begin{array}{l}32 \text { patients } \\
\text { with resistant } \\
\text { hypertension ( } 15 \\
\text { male/17 female, } \\
\text { mean age } 53.7 \pm \\
6 \text { yrs.) randomly } \\
\text { assigned to } \\
\text { HEx ( } n=16,8 \\
\text { male/8 female, } \\
\text { mean age } \\
55 \pm 5,9 \text { yrs.) or } \\
\text { CON ( } n=16.6 \\
\text { male/10f emale, } \\
\text { mean age } 52.4 \pm \\
5.9 \text { yrs.) groups. }\end{array}$ & $\begin{array}{l}60 \text { min of } \\
\text { moderate-intensity } \\
\text { (11-13 in the 6-20 } \\
\text { RPE) aerobic } \\
\text { (walking inside the } \\
\text { pool immersed } \\
\text { up to the xiphoid } \\
\text { process) and } \\
\text { callisthenic } \\
\text { exercise } \\
\text { (immersed up } \\
\text { to the xiphoid } \\
\text { process) } \\
\text { performed } 3 \text { times } \\
\text { a week. }\end{array}$ & NR & $\begin{array}{l}\text { The control } \\
\text { group was } \\
\text { asked to } \\
\text { maintain habitual } \\
\text { activities. }\end{array}$ & $32{ }^{\circ} \mathrm{C}$ & $\begin{array}{l}\text { Systolic and diastolic } \\
\text { BP were measured } \\
\text { before, and after } 2 \text { and } \\
12 \text { weeks of HEx and } \\
\text { CON intervention, using } \\
\text { a standard mercury } \\
\text { sphygmomanometer. } \\
\text { Ambulatory BP (systolic } \\
\text { and diastolic) was } \\
\text { measured during } 24 \text { h } \\
\text { before, and after } 2 \text { and } \\
12 \text { weeks of HEx and } \\
\text { CON intervention, using } \\
\text { an automatic device } \\
\text { (Spacelabs model). }\end{array}$ \\
\hline
\end{tabular}


(Conclusion)

\begin{tabular}{|c|c|c|c|c|c|c|}
\hline $\begin{array}{l}\text { First author } \\
\text { (Country) / } \\
\text { Design }\end{array}$ & $\begin{array}{l}\text { Population } \\
\text { assessed }\end{array}$ & HEx intervention & LEx intervention & $\begin{array}{l}\text { Control } \\
\text { intervention }\end{array}$ & $\begin{array}{l}\text { Water } \\
\text { temperature }\end{array}$ & $\begin{array}{l}\text { BP and secondary } \\
\text { outcomes assessment }\end{array}$ \\
\hline \multicolumn{7}{|c|}{ Studies assessing chronic adaptation to HEx } \\
\hline $\begin{array}{l}\text { Lambert et al. } \\
\text { (USA) / } \\
\text { Parallel [30] }\end{array}$ & $\begin{array}{l}60 \text { normotensive } \\
\text { subjects ( } 30 \\
\text { male/30 female) } \\
\text { randomly } \\
\text { assigned to } \\
\mathrm{HEx}(\mathrm{n}=36.19 \\
\text { male/17 female, } \\
\text { mean age } \\
41 \pm 2 \text { yrs.) } \\
\text { or } \mathrm{LEx} \text { ( } \mathrm{n}= \\
24.11 \text { male/13 } \\
\text { female, mean } \\
\text { age } 42 \pm 2 \text { yrs.) } \\
\text { groups. }\end{array}$ & $\begin{array}{l}\text { Exercise training } \\
\text { included three } \\
\text { sessions per } \\
\text { week for } 12 \\
\text { weeks with } \\
\text { sessions } \\
\text { progressively } \\
\text { increasing from } \\
250 \text { kcal per } \\
\text { session, } 60 \% \\
V^{*} 0_{2} \text { max during } \\
\text { the first week, } \\
\text { to } 500 \text { kcal per } \\
\text { session, } 85 \% \\
V^{\cdot} 0_{2} \text { max during } \\
\text { weeks } 6-12 \text {. }\end{array}$ & $\begin{array}{l}\text { Exercise training } \\
\text { included three } \\
\text { sessions per } \\
\text { week for } 12 \\
\text { weeks with } \\
\text { sessions } \\
\text { progressively } \\
\text { increasing from } \\
250 \text { kcal per } \\
\text { session, } 60 \% \text { V } \\
0_{2} \text { max during the } \\
\text { first week, to } 500 \\
\text { kcal per session, } \\
85 \% \text { V } 0_{2} \text { max } \\
\text { during weeks } \\
6-12 \text {. }\end{array}$ & NR & $33^{\circ} \mathrm{C}$ & $\begin{array}{l}\text { BP measured before, } \\
\text { at the end of each } \\
\text { stage, and for } 5 \\
\text { min after exercise } \\
\text { testing. Serial BP } \\
\text { measurements } \\
\text { were obtained } \\
\text { using standard } \\
\text { sphygmomanometric } \\
\text { procedures with } \\
\text { subjects resting in the } \\
\text { supine position during } \\
\text { the last } 30 \text { s of each } \\
3 \text {-min stage of the } \\
\text { graded exercise stress } \\
\text { tests and at } 1,3 \text {, and } 5 \\
\text { min during recovery. }\end{array}$ \\
\hline $\begin{array}{l}\text { Silva et al. } \\
\text { (Brazil) / } \\
\text { Parallel [31] }\end{array}$ & $\begin{array}{l}26 \text { hypertensive } \\
\text { subjects ( } 11 \\
\text { male/ } 15 \text { female) } \\
\text { randomly } \\
\text { assigned to } \\
\text { HEx ( } n=13.4 \\
\text { male/9 female, } \\
\text { mean age } 38.40 \\
\pm 8.24 \text { yrs.) or } \\
\text { CON ( } n=13.7 \\
\text { male/6 female, } \\
\text { mean age } 38.36 \\
\pm 8.96 \text { yrs.) } \\
\text { groups. }\end{array}$ & $\begin{array}{l}\text { Regular } \\
\text { swimming } \\
\text { program } \\
\text { consisting of } \\
\text { three weekly } \\
\text { fifty-minute } \\
\text { sessions of } \\
\text { training for } 10 \\
\text { weeks. The initial } \\
\text { sessions had } \\
\text { an intensity of } \\
\text { around } 40 \% \text { of } \\
\text { HRmax. }\end{array}$ & NR & $\begin{array}{l}\text { Did not change } \\
\text { his eating } \\
\text { habits and } \\
\text { remained without } \\
\text { practicing } \\
\text { physical activity } \\
\text { in the period. }\end{array}$ & $27-28^{\circ} \mathrm{C}$ & $\begin{array}{l}\text { BP was measured } \\
\text { with a digital arterial } \\
\text { pressure monitor } \\
\text { (Omrom } ® \text { HEM- } \\
741 \mathrm{C} \text { model, Japan). } \\
\text { The measurements } \\
\text { were taken always } \\
\text { at the same time of } \\
\text { day. Before training, } \\
\text { the subjects were at } \\
\text { rest for at least } 10 \\
\text { minutes prior to the } \\
\text { measurement of BP. } \\
\text { Soon after the training } \\
\text { session, the subjects } \\
\text { remained at rest again } \\
\text { for about } 10 \text { minutes } \\
\text { before measurement. }\end{array}$ \\
\hline $\begin{array}{l}\text { Silva et al. } \\
\text { (Portugal) / } \\
\text { Parallel [32] }\end{array}$ & $\begin{array}{l}36 \text { pre- } \\
\text { hypertensive } \\
\text { men randomly } \\
\text { assigned to HEx } \\
(\mathrm{n}=24, \text { mean } \\
\text { age } 40.60 \pm \\
9.36 \text { yrs.) or } \\
\text { CON ( }=12, \\
\text { mean age } 40.57 \\
\pm 8.05 \text { yrs.) } \\
\text { groups. }\end{array}$ & $\begin{array}{l}\text { A regular } \\
\text { swimming } \\
\text { program was } \\
\text { conducted, with } \\
\text { three weekly } \\
\text { sessions of } 45 \\
\text { minutes for } 12 \\
\text { weeks. The initial } \\
\text { sessions had } \\
\text { an intensity of } \\
\text { around } 40 \% \text { to } \\
50 \% \text { of HR max. }\end{array}$ & NR & $\begin{array}{l}\text { Did not change } \\
\text { his eating } \\
\text { habits and } \\
\text { remained without } \\
\text { practicing } \\
\text { physical activity } \\
\text { in the period. }\end{array}$ & $27-29^{\circ} \mathrm{C}$ & $\begin{array}{l}\text { BP was measured } \\
\text { according to the } \\
\text { recommendations } \\
\text { of the ACSM } \\
\text { hypertension } \\
\text { diagnosis, and was } \\
\text { measured using an } \\
\text { oscillometric device } \\
\text { (Omrom, model HEM- } \\
741 C \text {, Japan). }\end{array}$ \\
\hline
\end{tabular}

Note: Exercise in heated pool (HEx), Exercise in land based (LEx), Control without exercise (CON), Years (yrs.), Scale of 6-20 in perceived exertion (RPE), Pedaling Rate (rpm), Heart Rate (HR), Heart rate maximal (HRmax), Beats per minute (bpm), Blood Pressure (BP), American College of Sports Medicine (ACSM), Not reported (NR). 
Table 2 - PEDro score of the included studies

\section{Criteria PEDro}

\begin{tabular}{|c|c|c|c|c|c|c|c|c|c|c|c|c|}
\hline \multirow[b]{2}{*}{$\begin{array}{l}\text { First Author, } \\
\quad \text { Year }\end{array}$} & \multicolumn{12}{|c|}{ Criteria PEDro } \\
\hline & 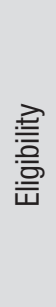 & 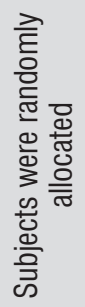 & 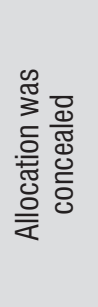 & 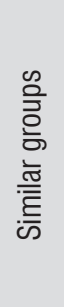 & 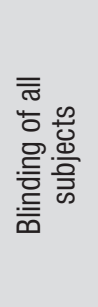 & 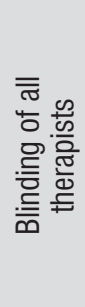 & 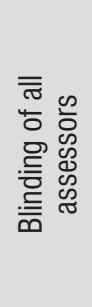 & 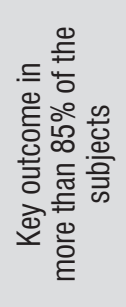 & 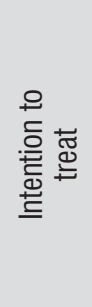 & 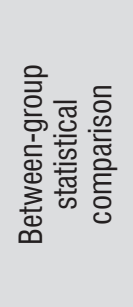 & 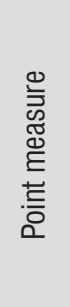 & Total \\
\hline Arca, 2013 [29] & $Y$ & $Y$ & $Y$ & $Y$ & $\mathrm{~N}$ & $\mathrm{~N}$ & $\mathrm{~N}$ & $Y$ & $Y$ & $Y$ & $Y$ & $7 / 10$ \\
\hline Castro, 2016 [6] & $Y$ & Y & Y & Y & N & $\mathrm{N}$ & Y & $Y$ & Y & Y & Y & $8 / 10$ \\
\hline $\begin{array}{l}\text { Colado, } 2009 \\
{[33]}\end{array}$ & Y & Y & $Y$ & Y & $Y$ & N & N & $\mathrm{N}$ & Y & Y & Y & $7 / 10$ \\
\hline $\begin{array}{c}\text { Cunha, } 2016 \\
\text { [28] }\end{array}$ & Y & Y & Y & Y & Y & $\mathrm{N}$ & N & N & Y & Y & Y & $7 / 10$ \\
\hline $\begin{array}{c}\text { Farahani, } 2010 \\
{[34]}\end{array}$ & Y & N & $\mathrm{N}$ & Y & N & $\mathrm{N}$ & N & N & Y & Y & Y & $4 / 10$ \\
\hline $\begin{array}{c}\text { Guimarães, } 2014 \\
\text { [18] }\end{array}$ & Y & Y & Y & Y & N & N & Y & $\mathrm{N}$ & Y & Y & Y & $7 / 10$ \\
\hline $\begin{array}{c}\text { Garzon, } 2014 \\
\text { [23] }\end{array}$ & Y & Y & Y & N & N & N & N & $Y$ & Y & Y & Y & $6 / 10$ \\
\hline $\begin{array}{c}\text { Lambert, } 2014 \\
{[30]}\end{array}$ & Y & Y & Y & Y & Y & N & Y & Y & Y & Y & Y & $9 / 10$ \\
\hline Luza, 2011 [26] & $\mathrm{Y}$ & Y & $\mathrm{N}$ & Y & Y & $\mathrm{N}$ & N & N & Y & Y & Y & $6 / 10$ \\
\hline Pugh, 2014 [27] & Y & Y & $\mathrm{N}$ & N & N & $\mathrm{N}$ & N & N & N & Y & Y & $3 / 10$ \\
\hline Silva, 2009 [31] & Y & Y & $Y$ & N & N & N & N & N & Y & Y & Y & $5 / 10$ \\
\hline Silva, 2015 [32] & Y & Y & N & Y & N & $\mathrm{N}$ & N & Y & Y & Y & Y & $6 / 10$ \\
\hline $\begin{array}{c}\text { Sosner, } 2016 \\
\text { [21] }\end{array}$ & Y & Y & $\mathrm{N}$ & Y & N & $Y$ & Y & $Y$ & Y & Y & Y & $8 / 10$ \\
\hline
\end{tabular}

Note: $\mathrm{Y}=$ yes; $\mathrm{N}=$ no.

\section{BP and secondary outcomes assessment}

The studies evaluating the acute and chronic effect of HEx measured BP by means of standard mercury sphygmomanometer[18,23,26,29,30,33,34],semiautomatic devices [28], brachial cuff-based oscillometric automatic device (24h) [6, 18, 21, 32], Finometer PRO to monitor BP beat-to-beat [27] and digital arterial pressure monitor [31].

\section{Characteristics of HEX}

The studies assessing the acute effect of HEx on BP reported low-intensity [27], moderate-intensity [6,26, 28], and high-intensity aerobic exercise sessions [21,23], with $30 \min [6,21,27]$ or $45 \mathrm{~min}[26,28]$ of duration; however, one study did not report exercise duration [23]. The water temperature ranged from $28.5^{\circ} \mathrm{C}$ to $32^{\circ} \mathrm{C}$. The studies assessing the chronic effect of HEx on BP reported lowintensity [31, 32] and moderate-intensity [17, 18, 29, 30] exercise sessions, with 35 to 60 min of duration; however, one study did notreport exercise session duration [30]. The exercise sessions were performed three times a week in six studies [17,18, 29-32], and progress from two to threetimes a week in one study [33]. The water temperature ranged from $27^{\circ} \mathrm{C}$ to $33.5^{\circ} \mathrm{C}$; however, the water temperature during HEx was not reported in one study [33].

\section{Effects of HEx on BP and secondary outcomes}

The main results and conclusions of acute and chronic water-based exercise on BP are described in Table 3. Five studies showed significant acute BP reduction (systolic and/or diastolic) after HEx [6, 21, 26-28]. On the other hand, one study did not observe 
acute hypotensive effects after HEx [23]. Considering the chronic effects, six studies showed significant BP reduction after HEx training when compared to control group and/or land-based exercise group [18, 29, 30, 32-34], and only one study showed no effect of HEx training in BP [31]. Indeed, one study observed similar $B P$ reduction in both HEx and LEx group, which were greater than in CON group [29]. Therefore, the results of this review demonstrated some divergent effects in chronic or acute approach post-HEx in BP.

Table 3 - Main results on blood pressure variables, other hemodynamic parameters, secondary measurements and the conclusions of the acute and chronic studies

(To be continued)

\begin{tabular}{|c|c|c|c|}
\hline $\begin{array}{l}\text { Studies assessing } \\
\text { the acute response } \\
\text { to heated water- } \\
\text { based exercise }\end{array}$ & & & \\
\hline Luza et al. [26] & $\begin{array}{l}\text { In the hypertensive group, the exercise protocol } \\
\text { on the ground caused an average reduction of } \\
16.5 \pm 3.7 \mathrm{mmHg}(\mathrm{P}=0.01) \text { in SBP at } 90 \\
\text { minutes post-exercise. In the normotensive } \\
\text { group, the protocol of rest in the water caused } \\
\text { an average reduction of } 14 \mathrm{bpm}(\mathrm{P}<0.01) \text { in } \\
\mathrm{HR} \text {. }\end{array}$ & $\begin{array}{l}\text { The volume of diuresis was } \\
\text { increased when compared with } \\
\text { the protocols accomplished on } \\
\text { the ground }(P<0.01) \text {. }\end{array}$ & $\begin{array}{l}\text { The results suggest that when } \\
\text { a physical exercise lasts } \\
45 \text { minutes, at submaximal } \\
\text { intensity, it causes reduction } \\
\text { of SBP in hypertensive } \\
\text { individuals. }\end{array}$ \\
\hline Pugh et al. [27] & $\begin{array}{l}\text { The HEx and LEx bouts were closely } \\
\text { matched for mean arterial BP in } 106 \mathrm{mmHg} \\
\text { ( } 95 \% \mathrm{Cl}) \text { vs } 101 \mathrm{mmHg}(95 \% \mathrm{Cl}) \text {, and } \\
\text { heart rate ( } 95 \mathrm{bpm}(95 \% \mathrm{Cl}, 90-101) \text { vs } 96 \\
\text { bpm ( } 95 \% \mathrm{Cl}, 91-102, \mathrm{P}=0.65) \text {. }\end{array}$ & $\begin{array}{l}\text { The oxygen consumption was similar } \\
\text { between the groups ( } 13.3 \mathrm{~mL} . \mathrm{kg} . \mathrm{min} \\
(95 \% \mathrm{Cl}, 12.2-14.6) \mathrm{vs} 13.5 \mathrm{~mL} . \mathrm{kg} \text {. } \\
\text { min ( } 95 \% \mathrm{Cl}, 12.1-14.8), \mathrm{P}=0.89) \text {. } \\
\text { Compared with land-based exercise, } \\
\text { water-based exercise induced an } \\
\text { increase in middle cerebral artery blood } \\
\text { flow velocity } 74 \mathrm{cms} \text { ( } 95 \% \text { confidence } \\
\text { interval, } 66-81) \text { vs } 67 \mathrm{cms}(95 \% \mathrm{Cl} \text {, } \\
\mathrm{P}=0.001) \text {, posterior cerebral artery } \\
\text { blood flow velocity } 47 \mathrm{cms}(95 \% \mathrm{Cl} \\
\text { ) vs } 43 \mathrm{~cm} \text { s (95\% } \mathrm{Cl}, \mathrm{P}=0.001) \text {, } \\
\text { and partial pressure of expired } \mathrm{CO}_{2}(\mathrm{P} \\
=0.001) \text {. }\end{array}$ & $\begin{array}{l}\text { Water based exercise induces } \\
\text { greater increase in cerebral blood } \\
\text { flow velocity than land-based } \\
\text { exercise of matched intensity. The } \\
\text { water immersion may enhance } \\
\text { the recurrent episodic increases } \\
\text { in cerebral blood flow and shear } \\
\text { stress that occur during exercise } \\
\text { and, subsequently, amplify } \\
\text { cerebrovascular health benefits } \\
\text { associated with exercise training. }\end{array}$ \\
\hline Cunha et al. [28] & $\begin{array}{l}\text { Overall ( } n=18) \text {, DBP did not change after the } \\
\text { HEx and CON, and SBP decreased at } 10 \text { and } \\
20 \text { min post exercise compared to the CON. } \\
\text { Among overweight women, SBP decreased at } \\
10 \text { and } 20 \text { minutes post exercise. In contrast, } \\
\text { among obese women, SBP decreased only at } \\
10 \text { minutes post exercise. SBP variation was } \\
2.68 \text { mmHg in overweight and } 2.4 \mathrm{mmHg} \text { in } \\
\text { obese women. }\end{array}$ & NA & $\begin{array}{l}\text { The HEx session leads to a } \\
\text { reduction in SBP, but not in } \\
\text { DBP, during } 10 \text { and } 20 \text { minutes } \\
\text { post exercise recovery. }\end{array}$ \\
\hline Castro et al. [6] & $\begin{array}{l}\text { No significant differences between interventions } \\
\text { were found in } 24 \mathrm{~h} \text { and nighttime BP. However, } \\
\text { daytime DBP was significantly lower after HEx } \\
\text { than CON }(-4 \pm 1.6 \mathrm{mmHg}, \mathrm{P}=0.03) \text {, and } \\
\text { daytime DBP tended to be lower after LEx than } \\
\mathrm{CON}(-2.3 \pm 1.1 \mathrm{mmHg}, \mathrm{P}=0.052) \text {. Hourly } \\
\text { analysis showed that SBP and DBP values } \\
\text { were lower after HEx (average reductions of } 6.6 \\
\text { to } 12.3 \mathrm{mmHg}, \mathrm{P}<0.01 \text { ) and LEx (average } \\
\text { reductions of } 5 \text { to } 8.3 \mathrm{mmHg}, \mathrm{P}<0.05) \text { than } \\
\text { after CON in several hours. No significant } \\
\text { differences between HEx and LEx were found in } \\
\text { any ambulatory BP data. }\end{array}$ & NA & $\begin{array}{l}\text { HEx and LEx promoted similar } \\
\text { reductions in ambulatory BP } \\
\text { of heart transplant recipients. } \\
\text { This post exercise hypotension } \\
\text { occurred even though the heart } \\
\text { transplant recipient patients had } \\
\text { lower control ambulatory BP } \\
\text { levels (average } 24 \mathrm{~h} \text { of } 122 / 81 \\
\text { mmHg), which does not occur } \\
\text { in non-heart transplant recipient } \\
\text { populations. These results } \\
\text { suggest that both exercises } \\
\text { may be tools to counteract } \\
\text { hypertension in this high-risk } \\
\text { population. }\end{array}$ \\
\hline
\end{tabular}


(To be continued)

\begin{tabular}{|c|c|c|c|}
\hline $\begin{array}{l}\text { Studies assessing } \\
\text { the acute respons } \\
\text { to heated water- } \\
\text { based exercise }\end{array}$ & & & \\
\hline Garzon et al. [23] & $\begin{array}{l}\mathrm{BP} \text { and systemic vascular resistance were } \\
\text { not different in exercise on HEx and LEx in } \\
\text { ergocycle. The stroke volume and cardiac } \\
\text { output were significantly higher during exercise } \\
\text { on immersible ergocycle ( } P<0.05, g=0.59 \\
\text { and } 0.20 \text { respectively). The stroke volume, } \\
\text { ejection fraction and contractility index were } \\
\text { higher ( } P<0.05, g=0.64,0.71 \text { and } 0.19 \\
\text { respectively). }\end{array}$ & $\begin{array}{l}\text { Both oxygen uptake and } \\
\text { arteriovenous difference were } \\
\text { significantly lower during exercise } \\
\text { on immersible ergocycle }(\mathrm{P}< \\
0.001, \mathrm{~g}=-0.25 \text { and }-0.87 \\
\text { respectively). During recovery, } \\
\text { oxygen uptake and arteriovenous } \\
\text { difference were significantly } \\
\text { reduced in water }(\mathrm{P}<0.001, \mathrm{~g} \\
=-0.41 \text { and }-0.67) \text {. }\end{array}$ & $\begin{array}{l}\text { During exercise and recovery } \\
\text { in immersion, arteriovenous } \\
\text { differences were reduced in } \\
\text { healthy young participants, while } \\
\text { stroke volume and cardiac output } \\
\text { were increased for the same } \\
\text { external power output. During the } \\
\text { recovery, central hemodynamic } \\
\text { responses remained higher in } \\
\text { immersible ergocycle. BP and } \\
\text { systemic vascular resistance } \\
\text { were not different between the } \\
\text { two conditions. }\end{array}$ \\
\hline Sosner et al. [21] & $\begin{array}{l}\text { Dryland high intensity interval exercise } \\
\text { induced a significant decrease in both SBP } \\
\text { and DBP measures during the } 24 \mathrm{~h} \text { period } \\
\text { (SBP: }-3.6 \pm 5.7 \mathrm{mmHg}, \mathrm{P}=.04 \text {; } \mathrm{DBP} \text { : } \\
-2.8 \pm 3.0 \mathrm{mmHg}, \mathrm{P}=.00 \text { ) and the daytime } \\
\text { period (SBP: }-4.4 \pm 7.5 \mathrm{mmHg}, \mathrm{P}=.046 \text {; } \\
\text { DBP: }-2.9 \pm 4.0 \mathrm{mmHg}, \mathrm{P}=.02 \text { ) but not } \\
\text { during the nighttime period. High intensity } \\
\text { interval exercise immersed resulted in a } \\
\text { greater decrease from baseline in systolic } \\
\text { measures during the } 24 \mathrm{~h} \text { period SBP: }-6.8 \pm \\
9.5 \mathrm{mmHg}, \mathrm{P}=.02) \text { and the daytime period } \\
\text { (SBP: }-7.5 \pm 11.2 \mathrm{mmHg}, \mathrm{P}=.03) \text {. There } \\
\text { was also a decrease in diastolic measures } \\
\text { during the } 24 \mathrm{~h} \text { period (DBP: }-3.0 \pm 4.5 \\
\mathrm{mmHg}, \mathrm{P}=.03 \text { ) and the daytime period } \\
\text { (DBP: }-3.9 \pm 4.5 \mathrm{mmHg}, \mathrm{P}=.006 \text { ). }\end{array}$ & NA & $\begin{array}{l}\text { In individuals with a baseline } \\
\text { office BP } \geq 130 / 85 \mathrm{mmHg} \text {, } \\
\text { the } 24 \mathrm{~h} \text { BP load decreased } \\
\text { significantly following a bout of } \\
\text { high intensity interval exercise } \\
\text { performed on a stationary } \\
\text { cycle in immersed condition. }\end{array}$ \\
\hline
\end{tabular}

\section{Studies assessing}

the chronic

response to heated

water-based

exercise

\begin{tabular}{|c|c|c|}
\hline Guimarães et al. [18] & 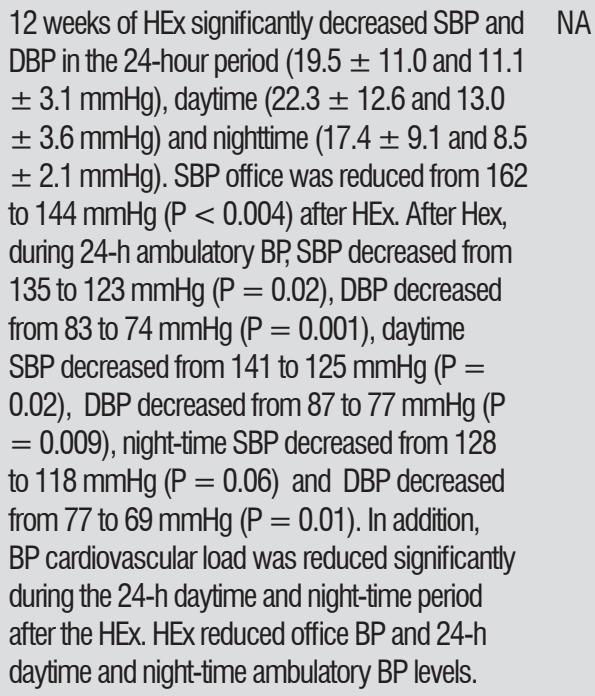 & $\begin{array}{l}\text { HEx induced beneficial effects } \\
\text { on BP in patients with resistant } \\
\text { hypertension, and there were } \\
\text { no signs of adverse reactions. } \\
\text { These effects suggest that } \\
\text { HEx may have a potential as a } \\
\text { new therapeutic approach to } \\
\text { resistant hypertensive patients. }\end{array}$ \\
\hline
\end{tabular}


(Conclusion

\begin{tabular}{|c|c|}
\hline $\begin{array}{l}\text { Studies assessing } \\
\text { the chronic response } \\
\text { to heated water- } \\
\text { based exercise }\end{array}$ & \\
\hline Arca et al. [29] & $\begin{array}{l}\text { Reduction on SBP in water group from } 136= \\
16 \mathrm{mmHg} \text { at week zero to } 124 \pm 15 \mathrm{mmHg} \\
\text { at week } 12 . \mathrm{In} \text { LEx, there was a reduction } \\
\text { from } 138 \pm 15 \mathrm{mmHg} \text { at week zero to } 126 \\
\pm 9 \mathrm{mmHg} \text { at week } 12 \text {. SBP has no change } \\
\text { in CON. }\end{array}$ \\
\hline Lambert et al. [30] & $\begin{array}{l}\text { HEx decreased resting DBP }(-3.2 \mathrm{mmHg}, \mathrm{P}< \\
0.05) \text { significantly when compared to LEx, SBF } \\
\text { (-9.0 to } 18.2 \mathrm{mmHg}, \mathrm{P}<0.05) \text {, mean arterial } \\
\text { pressure }(-4.8 \text { to } 8.3 \mathrm{mmHg}, \mathrm{P}<0.05) \text {, pulse } \\
\text { pressure }(-7.5 \text { to } 15 \mathrm{mmHg}, \mathrm{P}<0.05) \text {, and ra } \\
\text { pressure product ( }-1.8 \text { to } 3.9 \text { bpm.mmHg. } 103 \text {, } \\
\mathrm{P}<0.05) \text {. Under exercise stress, HEx, but not } \\
\mathrm{LEx} \text {, demonstrated reductions in } \mathrm{HR} \text { (range } \\
6.5-7.9 \text { bpm lower for each stage, } \mathrm{P}<0.05 \text { ). }\end{array}$ \\
\hline
\end{tabular}

Silva et al. [32]

The SBP and DBP significantly reduced only in HEx $(5.89 \mathrm{mmHg}$ for SBP and $5.15 \mathrm{mmHg}$ for DBP).

Silva et al. [31] Comparisons of the BP levels observed before and after the intervention revealed no statistically significant differences.

Colado et al. [33]

The DBP significantly reduced $(\mathrm{P} \leq 0.01)$ in both exercise groups $(6.8 \mathrm{mmHg}$ in HEx group and $4.8 \mathrm{mmHg}$ in the elastic band group).

Farahani et al. [34]

Exercise lowered SBP and mean arterial pressure by 11.71 (95\% confidence interval: 5.07 to 18.35$)$ and 5.90 (95\% confidence interval: 1.17 to 10.63) mmHg respectively. The lowering effect of exercise on DBP was neither statistically significant nor clinically important ( $0.55 \mathrm{mmHg} ; \mathrm{P}=0.8)$.
NA

NA

NA

NA

There was no significant effect of age, baseline body mass index and stage of hypertension on the exercise-induced changes in BP.
The lowering of BP in

hypertensive women submitted to HEx was similar to the one obtained with LEx and more intense than in the inactive CON. This controlled study addresses the antihypertensive effect of HEx in post-menopausal women.

The HEx preferentially ameliorates blood pressure reactivity to exercise stress, which would then be predicted to prevent or delay the onset of chronic diseases such as essential hypertension.

The results show that regular HEx causes significant decreases in systolic and diastolic pressures in prehypertensive men.

Despite evidence demonstrating the benefits of swimming for arterial pressure, it is necessary to emphasize the need for further studies to determine the optimal parameters for the prescription of physical activity for hypertensive individuals. Thus, the activities or forms of exercise can be selected to meet the personal characteristics of each individual in order to enhance the prevention, treatment, and control of hypertension.

Training with aquatic resistance exercises is a viable alternative to traditional resistance with elastic bands, and may provide more benefits to individuals who would be more sensitive to heavier loads or to impact, which may occur when training on LEx with certain devices and exercises.

A 10-week course of HEx markedly reduced the systolic and mean arterial BP of patients with essential hypertension and is especially recommended for the obese and the elderly who have orthopedic problems or bronchospasm.

Note: Exercise in heated pool (HEx), exercise in land based (LEx), Control group (CON), systolic blood pressure (SBP), diastolic blood pressure (DBP), blood pressure (BP), not assessment (NA), confidence interval (Cl). 


\section{Discussion}

The present systematic review evaluated the effect of HEx on BP in different populations. Although HEx failed to reduce BP levels after an acute [23] and a chronic [31] intervention study, the presentsystematic reviewidentified significant acute [6, 21, 26-28] and chronic [18, 29, 30, 32-34] hypotensive effect of HEx in different populations.

There are several physiological mechanisms triggered by the aquatic environment which benefits the neurohumoral control of BP $[22,35,36]$. The physical properties of water, such as the hydrostatic pressure, are responsible for facilitating venous return, which stimulates baroreceptors to trigger the increase in cardiac filling volume and stroke volume, reflexively reducing heart rate and $\mathrm{BP}[6,35-37]$. In addition, heated water ranging from 30 to $32^{\circ} \mathrm{C}$ triggers a reduction in peripheral vascular resistance due to dilatation of arterioles $[6,18,38]$.

Moreover, HEx reduces circulating levels of adrenergic neurotransmitters epinephrine and norepinephrine, renin and endothelin-1, as well as increases circulating levels of nitric oxide, which may results in reduced peripheral vascular resistance [38]. Regarding neurohumoral regulation, the improved renal system undergoes inhibition of renin-angiotensinaldosterone system, increases factors that excrete sodium (atrial natriuretic peptide), and favors diuresis, which reduce blood volume $[9,18,38]$. Other hypothesis is that there are improvements in sympathomodulatory and arterial baroreceptors after HEx, which reduce BP.

In contrast to the beneficial results of HEx, two studies did not identify differences in BP values in patients with hypertension [31] and healthy normotensive individuals [23]. These effects can be attributed to methodological differences in relation to training protocols, when compared with the other studies that found reduction of BP in the same populations [18, 21, 26-30,32]. One possible explanation for the lack of hypotensive effect in the above mentioned studies is that the exercise protocol did not follow the recommendations for dynamic exercises, such as an exercise intensity between $50 \%$ and $80 \%$ of peak oxygen consumption [2].

Muscle mass is another important factor that may contribute to the post-exercise hypotension after HEx. The larger muscle mass involved in HEx may result in larger production of vasodilatory agents, such as adenosine, potassium, lactate, nitric oxide and prostaglandin [24]. Although some land-based exercises (e.g. walking, running, cycling) require the participation of large muscle groups, it appears that HEx requires a greater activation of major muscle groups due to the resistance imposed by the water, thus triggering hypotension. In this context, heart failure patients who underwent an exercise program associating LEx and HEx (water temperature of $31^{\circ} \mathrm{C}$ ) showed substantial reduction in diastolic BP (11 $\mathrm{mmHg}$ ) after 24 weeks of training, which did not occur in patients who underwent LEx only for the same period [25]. This result supports the superior effects of HEx due to the mechanisms previously mentioned.

Therefore, the present systematic review identified a variety of training protocols that make it difficult to identify the best dose response of training to promote changes in BP effectively. However, HEx can be an optional exercise tool for reducing BP, isolated or in association with other exercise interventions. HEx also allows the practice of exercise for those individuals who are unable to perform LEx due to some physical limitation.

\section{Study limitations}

The present review was limited by the heterogeneity and degree of the pathological conditions among the studied populations, associated with the inclusion of both gender in the same samples, and the variety of training protocols, which made it impossible to carry out a meta-analysis.

\section{Conclusion}

Although negative findings exist, the present review suggests that HEx training performed for 12 to 24 weeks may reduce $\mathrm{BP}$ in normotensive, prehypertensive, hypertensive, postmenopausal women populations, whereas a HEx session may be efficient to acutely reduce $\mathrm{BP}$ in normotensive, hypertensive and heart transplant individuals. However, there is no homogeneity in the protocols used, which may have led to the heterogeneity in magnitude and duration of HEx-induced BP reductions.

\section{Acknowledgement}

This work is part of Awassi Y. Ngomane Master of Sciences dissertation under the guidance of Dr. Emmanuel G. Ciolac. Awassi Y. Ngomane was supported 
by Fundação de Amparo à Pesquisa do Estado de São Paulo (FAPESP \# 2015/05259-2) during this project.

\section{References}

1. Mancia G, Fagard R, Narkiewicz K, Redón J, Zanchetti A, Böhm M, et al. 2013 Practice guidelines for the management of arterial hypertension of the European Society of Hypertension (ESH) and the European Society of Cardiology (ESC): ESH/ESC Task Force for the Management of Arterial Hypertension. J Hypertens. 2013;31(10):1925-38.

2. James PA, Oparil S, Carter BL, Cushman WC, DennisonHimmelfarb C, Handler J, et al. 2014 evidence-based guideline for the management of high blood pressure in adults: report from the panel members appointed to the Eighth Joint National Committee (JNC 8). JAMA. 2014;311(5):507-20.

3. NCD Risk Factor Collaboration (NCD-RisC). Worldwide trends in blood pressure from 1975 to 2015: a pooled analysis of 1479 population-based measurement studies with 19.1 million participants. Lancet. 2017;389(10064):37-55.

4. Ciolac EG, Guimarães GV,D'Avila VM, Bortolotto LA, Doria EL, Bocchi EA. Acute effects of continuous and interval aerobic exercise on 24-h ambulatory blood pressure in long-term treatedhypertensive patients. IntJCardiol.2009;133(3):381-7.

5. Ciolac EG, Guimarães GV, D'Avila VM, Bortolotto LA, Doria EL, Bocchi EA. Acute aerobic exercise reduces 24-h ambulatory blood pressure levels in long-termtreated hypertensive patients. Clinics (São Paulo). 2008;63(6):753-8.

6. Castro RE, Guimarães GV, Silva JM, Bocchi EA, Ciolac EG. Postexercise Hypotensionafter HeartTransplant:Water-versus Land-Based Exercise.Med SciSportsExerc. 2016;48(5):804-10.

7. Pascoalino LN, Ciolac EG, Tavares AC, Castro RE, AyubFerreira SM, Bacal F, et al. Exercise training improves ambulatory blood pressure but not arterial stiffness in heart transplant recipients. J Heart Lung Transplant. 2015;34(5):693-700.

8. Guimarães GV, Ciolac EG, Carvalho VO, D'Avila VM, Bortolotto LA, Bocchi EA. Effects of continuous vs. interval exercise training on blood pressure and arterial stiffness in treated hypertension. Hypertens Res. 2010;33(6):627-32.
9. Cornelissen VA, Smart NA. Exercise training for blood pressure: a systematic review and metaanalysis. J Am Heart Assoc. 2013;2(1):e004473.

10. Ciolac EG, Bocchi EA, Bortolotto LA, Carvalho VO, Greve JMD, Guimarães GV. Effects of high-intensity aerobic interval training vs. moderate exercise on hemodynamic, metabolic and neuro-humoral abnormalities of young normotensive women at high familial risk for hypertension. Hypertens Res. 2010;33(8):836-43.

11. Cornelissen VA, Buys R, Smart NA. Endurance exercise beneficially affects ambulatory blood pressure: a systematic review and meta-analysis. J Hypertens. 2013;31(4):639-48.

12. Ciolac EG, Bocchi EA, Greve JM, Guimarães GV. Heart rate response to exercise and cardiorespiratory fitness of young women at high familial risk for hypertension: effects of interval vs continuous training. Eur J Cardiovasc Prev Rehabil. 2011;18(6):824-30.

13. Ciolac EG. High-intensity interval training and hypertension: maximizing the benefits of exercise? Am J Cardiovasc Dis. 2012;2(2):102-10.

14. Pescatello LS, Franklin BA, Fagard R, Farquhar WB, Kelley GA, Ray CA. American College of Sports Medicine position stand. Exercise and hypertension. Med Sci Sports Exerc. 2004;36(3):533-53.

15. Michalsen A, Lüdtke R, Bühring M, Spahn G, Langhorst $\mathrm{J}$, Dobos GJ. Thermal hydrotherapy improves quality of life and hemodynamic function in patients with chronic heart failure. Am Heart J. 2003;146(4):728-33.

16. Cider A, Schaufelberger M, Sunnerhagen KS, Andersson B. Hydrotherapy - a new approach to improve function in the older patient with chronic heart failure. Eur J Heart Fail. 2003;5(4):527-35.

17. Guimarães GV, Cruz LGB, Tavares AC, Dorea EL, Fernandes-Silva MM, Bocchi EA. Effects of short-term heated water-based exercise training on systemic blood pressure in patients with resistant hypertension: a pilot study. Blood Press Monit. 2013;18(6):342-5.

18. Guimaraes GV, Cruz LGB, Fernandes-Silva MM, Dorea EL, Bocchi EA. Heated water-based exercise training reduces 24-hour ambulatory blood pressure levels in resistant hypertensive patients: a randomized controlled trial (HEx trial). Int J Cardiol. 2014;172(2):434-41. 
19. Castro RE, Guimarães GV, Silva JM, Bocchi EA, Ciolac EG. Postexercise Hypotension after Heart Transplant: Water- versus Land-Based Exercise. Med Sci Sports Exerc. 2016;48(5):804-10.

20. Dimeo F, Pagonas N, Seibert F, Arndt R, Zidek W, Westhoff TH.Aerobic exercise reduces blood pressure in resistant hypertension. Hypertension. 2012;60(3):653-8.

21. Sosner P, Gayda M, Dupuy O, Garzon M, Lemasson C, Gremeaux V, et al. Ambulatory blood pressure reduction following high-intensity interval exercise performed in water or dryland condition. J Am Soc Hypertens. 2016;10(5):420-8.

22. Becker BE, Hildebrand K, Whitcomb RK, Sanders JP. Biophysiologic Effects of Warm Water Immersion. Int J Aquatic Res Edu. 2009;3:24-37.

23. Garzon M, Juneau M, Dupuy O, Nigam A, Bosquet L, Comtois A, et al. Cardiovascular and hemodynamic responses on dryland vs. immersed cycling. J Sci Med Sport. 2015;18(5):619-23.

24. Casonatto J, Goessler KF, Cornelissen VA, Cardoso JR, Polito MD. The blood pressure-lowering effect of a single bout of resistance exercise: A systematic review and meta-analysis of randomised controlled trials. Eur J Prev Cardiol. 2016;23(16):1700-14.

25. 25. Corso LM, Macdonald HV, Johnson BT, Farinatti $\mathrm{P}$, Livingston J, Zaleski AL, et al. Is Concurrent Training Efficacious Antihypertensive Therapy? A Meta-analysis. Med Sci Sports Exerc. 2016;48(12):2398-406.

26. Luza M, Siqueira LO, Paqualotti A, Reolão JBC, Schmidt $\mathrm{R}$, Calegari L. Efeitos do repouso e do exercício no solo e na água em hipertensos e normotensos. Fisioter Pesqui. 2011;18(4):346-52.

27. Pugh CJ, Sprung VS, Ono K, Spence AL, Thijssen DH, Carter $\mathrm{HH}$, et al. The effect of water immersion during exercise on cerebral blood flow. Med Sci Sports Exerc. 2015;47(2):299-306.

28. Cunha RM, Arsa G, Neves EB, Lopes LC, Santana F, Noleto MV, et al. Water aerobics is followed by shorttime and immediate systolic blood pressure reduction in overweight and obese hypertensive women. J Am Soc Hypertens. 2016;10(7):570-7.

29. Arca EA, Martinelli B, Martin LC, Waisberg CB, Franco RJ. Aquatic exercise is as effective as dry land training to blood pressure reduction in postmenopausal hypertensive women. Physiother Res Int. 2014;19(2):93-8.
30. Lambert BS, Greene NP, Carradine AT, Joubert DP, Fluckey JD, Riechman SE, et al. Aquatic treadmill training reduces blood pressure reactivity to physical stress. Med Sci Sports Exerc. 2014;46(4):809-16.

31. Silva J, Geraldes A, Natali A, Pereira J, Vale R, Dantas E. Acute Effects of Swimming on the Arterial Pressure of Hypertensive Adults. Maced J Med Sci. 2009;2(4):330-4.

32. Silva JE, Teixeira AMB, Dantas EHM, Rama LMPL. Comportamento da pressão arterial em homens préhipertensos participantes em um programa regular de natação. Rev Bras Med Esporte. 2015;21(3):178-81.

33. Colado JC, Triplett NT, Tella V, Saucedo P, Abellán J. Effects of aquatic resistance training on health and fitness in postmenopausal women. Eur J Appl Physiol. 2009;106(1):113-22.

34. Farahani AV, Mansournia MA, Asheri H, Fotouhi A, Yunesian M, Jamali M, et al. The effects of a 10-week water aerobic exercise on the resting blood pressure in patients with essential hypertension. Asian J Sports Med. 2010;1(3):159-67.

35. Meredith-Jones K, Waters D, Legge M, Jones L. Upright water-based exercise to improve cardiovascular and metabolic health: a qualitative review. Complement Ther Med. 2011;19(2):93-103.

36. Rodriguez D, Silva V, Prestes J, Rica RL, Serra AJ, Bocalini DS, et al. Hypotensive response after water-walking and land-walking exercise sessions in healthy trained and untrained women. Int J Gen Med. 2011;4:549-54.

37. Ciolac EG, Castro RE, Greve JM, Bacal F, Bocchi EA, Guimaraes GV. Prescribing and Regulating Exercise with RPE after Heart Transplant: A Pilot Study. Med Sci Sports Exerc. 2015;47(7):1321-7.

38. Cruz LG, Bocchi EA, Grassi G, Guimaraes GV. Neurohumoral and Endothelial Responses to Heated Water-Based Exercise in Resistant Hypertensive Patients. Circ J. 2017;81(3):339-45.

Received in 05/29/2017

Recebido em 29/05/2017

Approved in 03/01/2018

Aprovado em 01/03/2018 